\title{
Search for fourth sound propagation in supersolid ${ }^{4} \mathrm{He}$
}

\author{
Y. Aoki ${ }^{1}$, X. Lin ${ }^{2}$, and H. Kojima ${ }^{1}$ \\ ${ }^{1}$ Serin Physics Laboratory, Rutgers University, Piscataway, NJ 08854, USA \\ E-mail: kojima@physics.rutgers.edu \\ ${ }^{2}$ Department of Physics, Pennsylvania State University, University Park, PA 16802, USA
}

Received October 18, 2007

\begin{abstract}
A systematic study was carried out to search for fourth sound propagation solid ${ }^{4} \mathrm{He}$ samples below $500 \mathrm{mK}$ down to $40 \mathrm{mK}$ between 25 and 56 bar using the techniques of heat pulse generator and titanium superconducting transition edge bolometer. If solid ${ }^{4} \mathrm{He}$ is endowed with superfluidity below $200 \mathrm{mK}$ as indicated by recent torsional oscillator experiments, theories predict fourth sound propagation in such supersolid state. If found, fourth sound provides a convincing evidence for superfluidity and a new tool for studying the new phase. To search for a fourth sound-like mode, the response of the bolometers to heat pulses traveling through cylindrical samples of solids grown with different crystal qualities. Bolometers with increasing sensitivity were constructed. The heater generator amplitude was reduced to the sensitivity limit to search for any critical velocity effects. The fourth sound velocity is expected to vary as $\propto \sqrt{\rho_{s} / \rho}$. Searches were made for signature in the bolometer response with such characteristic temperature dependence. The measured response signal has not so far revealed any signature of new propagating mode within the temperature excursion of $5 \mu \mathrm{K}$ from the background signal shape. Possible reasons for this negative result are discussed. Prior to the fourth sound search, the temperature dependence of heat pulse propagation was studied as it transformed from «second sound» in the normal solid ${ }^{4} \mathrm{He}$ to the transverse ballistic phonon propagation. Our work extended the studies by Narayanamurti and Dynes [Phys. Rev. B12, 1731 (1975)] to higher pressures and to lower temperatures. The measured transverse ballistic phonon propagation velocity remained constant, within $0.3 \%$ scatter of data below $100 \mathrm{mK}$ at all pressures and revealed no indication for an onset of supersolidity. The overall dynamic thermal response of solid to heat input was found to depend strongly on the sample preparation procedure.
\end{abstract}

PACS: 62.20.-x Mechanical properties of solids;

63.20.-e Phonons in crystal lattices;

67.80.-s Quantum solids.

Keywords: supersolid, fourth sound, second sound, ballistic phonon, bolometer, heat pulse.

\section{Introduction}

Numerous experiments [1] were attempted to search for superfluidity in solid ${ }^{4} \mathrm{He}$ that had been suggested to emerge theoretically by Andreev and Lifshitz [2] and Leggett [3]. The solid state with coexisting crystallinity and superfluidity has been called «supersolidity». No positive evidence for supersolidity had been reported until the recent torsional oscillator experiments by Kim and Chan $[4,5]$ which showed evidence of «non-classical rotational inertia» (NCRI) below about $200 \mathrm{mK}$ in solid ${ }^{4} \mathrm{He}$. These and other corroborative experiments [6-8] apparently indicated the existence of supersolid phase in solid ${ }^{4} \mathrm{He}$. Rittner and Reppy [8] found that the NCRI signal could be much reduced by annealing of the solid ${ }^{4} \mathrm{He}$ at $26 \mathrm{bar}$, and their observation showed the importance of sample preparation procedure. Day and Beamish $[9,10]$ measured pressure-driven flows of solid helium in Vycor pores and in bulk at low temperatures, and they did not see evidence for superflow as expected for a conventional superfluid system such as superfluid ${ }^{4} \mathrm{He}$ or He II. Sasaki et al. [11], on the other hand, observed evidence for a superfluid-like flow through a solid ${ }^{4} \mathrm{He}$ plug which was formed in a tube in contact with superfluid liquid ${ }^{4} \mathrm{He}$. The superfluid flow occurred when the solid plug contained large visible «cracks» but not when cracks disappeared. Recent measurements of heat capacity of solid ${ }^{4}$ He show deviations from the $T^{3}$ dependence of specific heat below the supersolid transition and support the pres- 
ence of a thermodynamic phase transition [12]. A precision measurement of the melting pressure at very low temperatures, however, does not show anomaly near the supersolid transition temperature [13].

In view of these varied and some conflicting experimental observations, the nature and the physical origin of the supersolid state have not been clarified yet and are currently under intense experimental and theoretical investigations [14]. The motivation for our experiments with heat pulse propagation in solid ${ }^{4} \mathrm{He}$ is to search for a new experimental probe for studying the properties of supersolid ${ }^{4} \mathrm{He}$. Theoretical studies $[2,15,16]$ indicate that there should appear new propagating modes such as fourth sound in the supersolid state just as they do in liquid ${ }^{4} \mathrm{He}$ [17] and ${ }^{3} \mathrm{He}$ [18] below their respective superfluid transition temperatures. Observation of these new modes would support the interpretation of the results of torsional oscillator experiments in terms of superfluidity of solid ${ }^{4} \mathrm{He}$. Our experiments may also shed light on the issue of how the supersolid behavior is affected by the existence of dislocations, impurities, defects, or grain boundaries in solid ${ }^{4} \mathrm{He}$ samples.

\section{Heat pulse and fourth sound propagations in solid ${ }^{4} \mathrm{He}$}

\subsection{Heat pulse and second sound propagation in normal solid ${ }^{4} \mathrm{He}$}

The manner of heat pulse propagation in solid ${ }^{4} \mathrm{He}$ evolves as a function of temperature $(T)$ depending on the characteristic time scale $(\tau)$ of the heat pulse. Let the relaxation time in the momentum conserving «normal» phonon collisions be $\tau_{n}(T)$ and that in the momentum nonconserving Umklapp collisions be $\tau_{u}(T)$. If $\tau>\tau_{u}$ as it occurs usually at high temperatures, the heat pulse «propagation» proceeds by diffusion. If $\tau<\tau_{n}$ as it might occur below a sufficiently low temperature, the heat pulse propagates ballistically. Ballistic phonon propagations have been observed in other dielectric materials such as quartz and sapphire by Gutfeld and Nethercot [19] and in silicon [20]. Narayanamurti and Dynes [21] observed tehm in both solid ${ }^{4} \mathrm{He}$ and ${ }^{3} \mathrm{He}$ at their respective melting pressures. Balibar [22] also studied ballistic phonon propagation phenomena in solid ${ }^{4} \mathrm{He}$. Our study here extended theirs in solid ${ }^{4} \mathrm{He}$ to measure the temperature dependence in much more detail and pressure dependence up to 56 bar.

In the intermediate restrictive temperature range, where $\tau_{n}<\tau<\tau_{u}$, heat pulse may propagate as a temperature wave or so-called «second sound» [23] in analogy with the temperature wave propagation in He II. Under this restrictive condition, the phonons have sufficient time to thermalize but have not yet suffered irreversible Umklapp collisions. The second sound propagation as de- fined here in a dielectric solid is then a propagation of collective oscillation in phonon density. It has been observed in solid ${ }^{4} \mathrm{He}$ and ${ }^{3} \mathrm{He}$ [24-26], sodium fluoride [27-29] and bismuth [30].

The crossover in the heat pulse propagation from second sound to ballistic phonon propagation in the dielectric materials was studied by Rogers [27]. A hydrodynamic description of the crossover was given by Rogers [27] and derived a dispersion relation given by

$$
k^{2} v_{\mathrm{II}}^{2}=\omega^{2}+\frac{i \omega k^{2} \tau_{n}\left(v_{\mathrm{I}}^{2}-v_{\mathrm{II}}^{2}\right)}{1-i \omega \tau_{n}} .
$$

Here, $v_{\mathrm{I}}$ and $v_{\mathrm{II}}$ are the velocities of ballistic propagation and second sound, respectively, and $\omega$ is a characteristic frequency. We found it convenient to apply Eq. (1) for analysis of our crossover data.

The second sound propagation in normal solid ${ }^{4} \mathrm{He}$ was first observed [25] and studied by Ackerman and Guyer [26]. They measured the changes in temperature pulse shape from diffusion to second sound wave propagation in solid ${ }^{4} \mathrm{He}$ up to $130 \mathrm{bar}$, and determined the second sound velocity and the normal phonon scattering relaxation time from the broadening of the temperature pulse. Later, Narayanamurti and Dynes [21] extended heat pulse propagation studies at the melting pressure down to $60 \mathrm{mK}$ to measure the propagation velocity in the ballistic regime. These previous experiments were not designed to search for evidence of supersolidity and did not include detailed measurements of temperature dependence below $100 \mathrm{mK}$. In a more recent work, the effect of second sound generated in normal solid ${ }^{4} \mathrm{He}$ in the propagation path of ultrasound was studied [31] to explore the previously observed anomaly [32] near $200 \mathrm{mK}$ in another ultrasound experiment. In our work, we carried out detailed studies of the crossover behavior in the heat pulse propagation from second sound to ballistic propagation as well as the temperature range entering deep into the ballistic regime at pressures between 25 and 56 bar with the explicit aim of finding new evidence for supersolidity.

\subsection{Fourth sound and hydrodynamics of supersolid ${ }^{4} \mathrm{He}$}

The hydrodynamics of supersolid state has been investigated theoretically by Andreev and Lifshitz [2], Saslow $[15,33]$ and Liu [16]. As in superfluid ${ }^{4} \mathrm{He}$, these theoretical works develop a two-fluid model with normal (density $\rho_{n}$, velocity $\left.v_{n}\right)$ and superfluid $\left(\rho_{s}, v_{s}\right)$ components which move independently in the presence of background lattice site motion. The hydrodynamics is more complicated than in He II since the lattice strain, $w_{i j}$, must be included with the usual two variables (say, the mass and entropy densities, $\rho$ and $s$, respectively) to specify the thermodynamic state. Saslow [33] emphasizes that the 
lattice must be assigned its own density $\left(\rho_{L}\right)$ and velocity $\left(v_{L}\right)$ which is usually set equal to $v_{n}$. The hydrodynamics produces three propagating modes, two longitudinal and one transverse. The two longitudinal modes are referred to as first (subscript $l$ ) and fourth (subscript 4) sound with propagation velocities, $c_{l}$ and $c_{4}$, respectively, given [34] (for simplicity, formulas are given for an isotropic solid) in the limit of $(1 / \rho)\left(\partial \rho / \partial w_{11}\right) \approx-1$ and a small superfluid fraction $\rho_{s} / \rho$ by:

$$
\begin{aligned}
& c_{l}^{2}=c_{l 0}^{2}\left(1+\frac{\rho_{s}}{\rho}\right), \\
& c_{4}^{2}=\rho_{s}\left(\frac{\partial \mu}{\partial \rho}\right)_{s, w} .
\end{aligned}
$$

The transverse mode velocity is given by:

$$
c_{t}^{2}=\frac{\rho}{\rho_{n}} c_{t 0}^{2} \approx c_{t 0}^{2}\left(1+\frac{\rho_{s}}{\rho}\right) .
$$

Here, $\rho$ is the total density, $c_{l 0}$ the longitudinal sound velocity in the normal solid, $\mu$ the chemical potential, and $c_{t 0}$ the transverse sound velocity in the normal solid.

There is no confusion about the first sound designation as it is analogous to the ordinary sound propagation in superfluid ${ }^{4} \mathrm{He}$. It can be shown [34] for the fourth sound mode propagation that $v_{n} \approx\left(\rho_{s} / \rho\right)^{2} v_{s}$ : the normal fluid moves little in this mode. In analogy to calling just such mode in He II as «fourth» sound, this mode in supersolid is referred to as such. It «transforms» to the second sound in superfluid ${ }^{4} \mathrm{He}$ in the limit of disappearance of crystallinity [16].

It is seen from Eqs. (2), (3), and (4) that supersolidity would be marked by changes in the propagation velocity of all modes. The goal of our experiments in this report is to search for such marks as new evidence for superfluidity in solid ${ }^{4} \mathrm{He}$. The propagation velocities of both the longitudinal and transverse modes are expected to increase by a detectable factor $\sim\left(1+\frac{1}{2} \rho_{s} / \rho\right)$. An unequivocal proof of superfluidity $[35,36]$ of matter would be the observation of fourth sound mode. Its propagation velocity is expected to directly reflect the superfluid density and to vanish at the transition temperature. The measurement of fourth sound velocity gives a measure of the superfluid fraction via Eq. (3). Frequency dependent effects may be more conveniently studied with fourth sound. If the attenuation can be measured, dissipative mechanisms in the superfluid state may be explored.

How might the fourth sound be best excited and detected? Consider first an acoustic transducer which produces motion in both the normal and superfluid components such that $v_{s}=v_{n}$ and total oscillating density amplitude $\rho^{\prime}=\rho\left(v_{n} / c_{l 0}\right)$. It can be shown [34] that the input energy from the transducer divides into first and fourth sounds according to:

$$
\begin{aligned}
& \frac{v_{n 4}}{v_{n l}}=\left(\frac{\rho_{s}}{\rho}\right)^{2}, \\
& \frac{\rho_{l}^{\prime}}{\rho}=\frac{v_{n l}}{c_{l 0}}, \\
& \frac{\rho_{4}^{\prime}}{\rho}=\left(\frac{\rho_{s}}{\rho}\right)^{1 / 2} \frac{\rho_{l}^{\prime}}{\rho} .
\end{aligned}
$$

If we let the transducer produce $v_{n l}=10 \mu \mathrm{m} / \mathrm{s}$ so as not to suppress the superfluid density [37], then the relative density oscillation in the first sound is $\rho^{\prime} / \rho \approx 2 \cdot 10^{-8}$ assuming $c_{l 0} \approx 500 \mathrm{~m} / \mathrm{s}$. If the superfluid fraction is $1 \%$ [38], Eq. (6) implies fourth sound excitation with the pressure amplitude of $6 \cdot 10^{-3} \mathrm{~Pa}$ in a 30 bar solid. This might be detectable with a piezoelectric microphone. Consider next a heater as a generator of fourth sound. If the heater produces no net mass flow as expected, it can be shown [34] that $v_{n 4} / v_{n l} \approx-\rho_{s} / \rho$. This appears more favorable in comparison to Eq. (5). We chose to attempt a heater/bolometer as the genertor/detector combination in search of fourth sound.

Dorsey, et al. [39] developed a phenomenological Landau theory of phase transition and considered the coupling of superfluidity to elasticity of the crystalline lattice. They predicted that the longitudinal sound velocity in single crystal samples should show an anomaly in its temperature dependence at the transition temperature when certain requirements on the polarization of sound are satisfied. Even if the sample is polycrystalline but isotropic, they predict that the velocity should vary as $\left|\left(T-T_{c}\right) / T_{c}\right|^{-\alpha}$ where $\alpha=-0.0127$. On the other hand, they also point out that local variations in elastic constants may lead to smearing out of the transition. This effect might make observing the predicted temperature dependence problematic.

\section{Experimental techniques}

The sample cell in this experiment was the same as that described in detail in our preliminary report [40]. Briefly, the sample chamber was made of copper containing a packed copper powder as heat exchanger and was attached to the mixing chamber. A resistive heater and a Ti superconducting transition edge bolometer detector were held apart vertically by spacers within the chamber (see below). The basic measurement was to detect the temporal development of temperature at the bolometer following a short current pulse applied to the heater. There was a «background» thermal relaxation of the whole solid ${ }^{4} \mathrm{He}$ in the sample chamber that occurred by the energy flow through the thermal boundary resistance of this heat exchanger subsequent to the applied heat pulses for generating fourth sound. A small superconducting solenoid magnet, thermally anchored to the continuous heat exchanger 
of the dilution refrigerator, was mounted around the sample chamber. The magnetic field was adjusted to optimize the operating point by shifting the transition temperature of the Ti bolometer.

Solid ${ }^{4} \mathrm{He}$ samples were grown either at constant pressure near 25.5 bar or at constant volume by the blocked capillary method at pressures up to 56 bar. To facilitate the blocked capillary method, the thermal contacts between the fill line (inner diameter $0.13 \mathrm{~mm}$ ) and the refrigerator below the still temperature were weakly made, and heaters were attached near the thermal contact locations. These heaters were turned on while introducing liquid into the cell and then off during formation of a block. The pressure in the cell was monitored by a capacitance pressure gauge attached to the same stage as the sample cell. Temperature of the solid was measured by a calibrated ${ }^{3} \mathrm{He}$ melting pressure thermometer and an auxiliary carbon resistance thermometer.

Heat pulses were generated by passing a current into a metallic film deposited onto a $(0.3 \mathrm{~mm}$ thick $5 \mathrm{~mm}$ square) silicon wafer, which was mounted on the upper wall of the cell. The heater film was $25 \mathrm{~nm}$ thick and 12 $\mu \mathrm{m}$ wide silver strips covering over a $40 \mathrm{~nm}$ thick Ti underlayer deposited with the same width for improving adherence. The effective area of the heater was $2.5 \times 3 \mathrm{~mm}$, and the resistance was $30 \Omega$ at $T \leq 1 \mathrm{~K}$. The heater currents were applied during short time $(t)$ intervals $0<t<t_{p w}$, where the current pulse width $t_{p w}$ was varied between 0.5 and $10 \mu$ s. The shape of the applied heater current was given by $I_{H} \sin { }^{2}\left(\pi t / t_{p w}\right)$, where $I_{H}(<25 \mathrm{~mA})$ was the current amplitude. The total heater energy input per pulse was varied over wide range between $10 \mathrm{pJ}$ and $10 \mathrm{~nJ}$. For our search for evidence of delicate effects arising from supersolidity, the input energy was reduced to much smaller levels than those in Ref. 21 where $6.1 \mathrm{~nJ} /$ pulse had been applied. A detector bolometer (see below) was also deposited onto a silicon wafer of the same dimensions as the heater substrate. The bolometer substrate was affixed to a brass plate which was suspended from the upper wall [40] by two brass posts. The distance $L$ between the heater and the bolometer was $4.3 \mathrm{~mm}$. The heater and the bolometer substrates were parallel to better than $0.3^{\circ}$.

Results obtained from two different Ti film bolometers are described in this paper. The first bolometer was the low resistance bolometer (LRB) that had been described in our previous report [40]. The LRB was a single strip of Ti film (60 nm thick, $0.5 \mathrm{~mm}$ wide and $3 \mathrm{~mm}$ long). The resistance $R$ of the film was $169 \Omega$ at $300 \mathrm{~K}$ and $106 \Omega$ at $1 \mathrm{~K}$. The DC bias current in the bolometer was adjusted around $5 \mu \mathrm{A}$ to optimize sensitivity and stability. The sensitivity, $d V / d T$, where $V$ is the voltage across the bolometer, was $25 \mu \mathrm{V} / \mathrm{mK}$ at the midpoint of its resistive transition at $450 \mathrm{mK}$. The bolometer could be magnetically biased to shift the midpoint to lower temperatures.
The sensitivity decreased to $0.5 \mu \mathrm{V} / \mathrm{mK}$ at $40 \mathrm{mK}$ in a magnetic field of $3.3 \mathrm{kG}$. The second high resistance bolometer (HRB) was Ti film deposited in a meander pattern: see Table 1 for its characteristics. The width of the bolometer film was $1.6 \mu \mathrm{m}$ and the thickness was $37 \mathrm{~nm}$. The meander pattern had about 25 turns (total length $7.5 \mathrm{~cm}$ ) within an effective area of $1.5 \times 3 \mathrm{~mm}$. Owing to its reduced film thickness, the transition edge in zero magnetic field was suppressed to $\sim 250 \mathrm{mK}$. A magnetic field of $1.3 \mathrm{kG}$ was applied to lower the operating point down to $40 \mathrm{mK}$. With the typical DC bias current of $1.5 \mathrm{nA}$ applied to the bolometer, the power dissipated in the bolometer during measurement was $0.36 \mathrm{pW}$. Measured resistance of HRB is shown in Fig. 1 as a function of temperature. The sensitivity of HRB was $6 \mu \mathrm{V} / \mathrm{K}$ with an operating current of $1.5 \mathrm{nA}$ at the midpoint of its resistive transition at $40 \mathrm{mK}$, and it was more than 10 times higher than that of LRB. Rounding of superconducting transition edge in Fig. 1 was likely caused by impurities and/or irregularities in the Ti film. A drawback of HRB was that, owing to its large resistance, the electronic RC time constant was long $(\sim 0.1 \mathrm{~ms})$. We used LRB for high precision heat pulse velocity measurement requiring short response times. For fourth sound search, HRB with higher sensitivity was more advantageous.

Table 1. Physical characteristics of bolometers.

\begin{tabular}{|c|c|c|c|c|c|c|}
\hline $\begin{array}{l}\overline{0} \\
\frac{0}{0} \\
\frac{0}{0} \\
0 \\
0\end{array}$ & 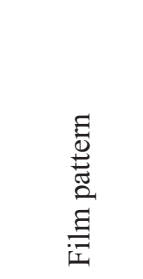 & $\begin{array}{l}\mathrm{C} \\
\hat{\sigma} \\
8 \\
\stackrel{0}{0} \\
\stackrel{0}{2}\end{array}$ & $\begin{array}{l}\mathrm{G} \\
\hat{\underline{\Omega}} \\
\stackrel{\Xi}{\Xi}\end{array}$ & $\begin{array}{l}v \\
0\end{array}$ & 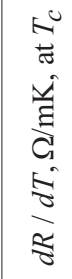 & 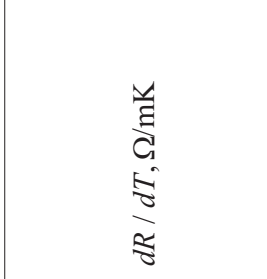 \\
\hline LRB & single strip & 169 & 106 & 0.45 & 5 & $0.1(40 \mathrm{mK}, 3.3 \mathrm{kG})$ \\
\hline HRB & meander & $10^{6}$ & $3.2 \cdot 10^{5}$ & 0.25 & $10^{4}$ & $4 \cdot 10^{3}(40 \mathrm{mK}, 1.3 \mathrm{kG})$ \\
\hline
\end{tabular}

\section{Results and discussions}

\subsection{Velocity of heat pulse propagation}

We first present our results on the heat pulse propagation obtained mostly with LRB. Measurements were made to observe the crossover from second sound in normal solid ${ }^{4} \mathrm{He}$ at high temperature to ballistic phonon propagation at lower temperature. We confirmed the results of the second sound propagation by earlier investigators [26] and extended the low temperature measurements [21] to pressures higher than the melting pressure. We focused our attention on the temperature range below $300 \mathrm{mK}$, where NCRI effects had been detected in torsion oscillator experiments. Our careful and systematic searches have not uncovered so far any signature which could be associated with NCRI at any temperature in any of our 


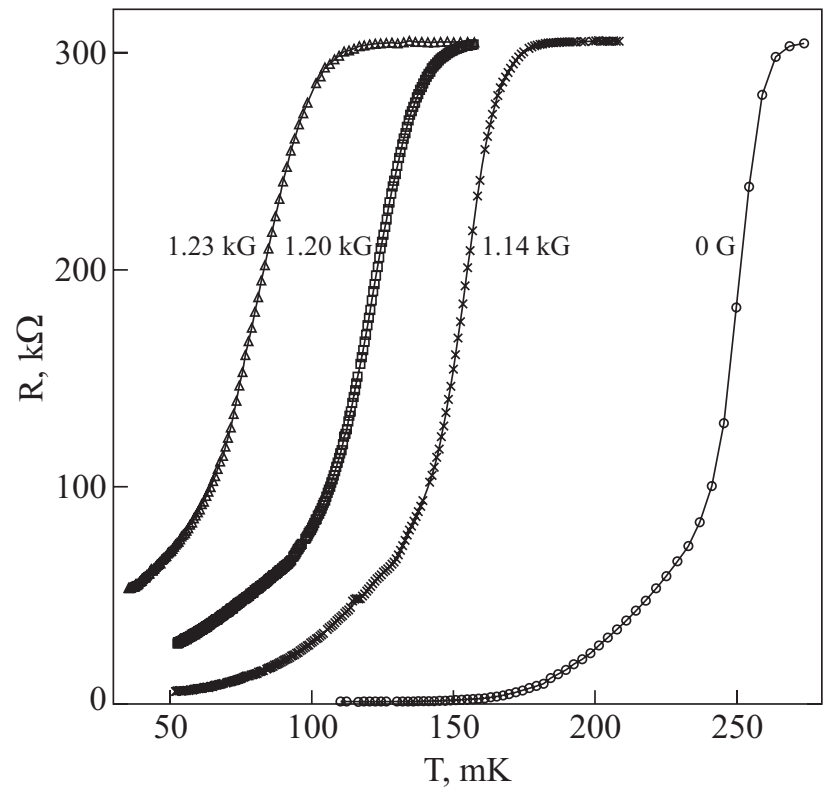

Fig. 1. Resistance vs. temperature of high resistance bolometer under bias magnetic field. In fields $1.2 \mathrm{kG}$ and below, the applied current was $4.6 \mathrm{nA}$, and in $1.23 \mathrm{kG}$ it was reduced to 0.9 nA.

solid samples between 26 and 56 bar. The drive level was varied and minimized to the extent allowed by sensitivity to search for effects related to critical velocity. Different procedures for preparing solid ${ }^{4} \mathrm{He}$ sample were attempted to see if some solid quality change might reveal anomalous behavior [8]. Though the propagation velocity did not depend on drive levels or on preparation procedures, the thermal equilibration time did.

Figure 2 shows the time dependence of the signal developed across LRB at representative temperatures. The solid sample here was grown with the blocked capillary technique and cooled to $1 \mathrm{~K}$ in about ten hours. To highlight the initial behavior, the time span only between 10 and $35 \mu$ s following the application of heat pulse is shown.

Thanks to the short response time of the bolometer, the signal shown in Fig. 2 is proportional to the temperature deviation from the ambient of the solid ${ }^{4} \mathrm{He}$ adjacent to the bolometer. At temperature higher than about $350 \mathrm{mK}$, the input heat pulses produced well-defined «propagating temperature pulses» but with widths much longer than $t_{p w}$ [41]. When the measurement time interval was extended, echo-like response (see Fig. 3) with reduced amplitude could be observed. At lower temperatures, «temperature pulses» lost the symmetrical shape seen at higher temperatures but the arrival signal edge became much sharper. After reaching the maximum response, the temperature deviation remained there over the time range shown in Fig. 2.

The observed temperature dependence of the signal response shown in Fig. 2 may be understood as the cross-

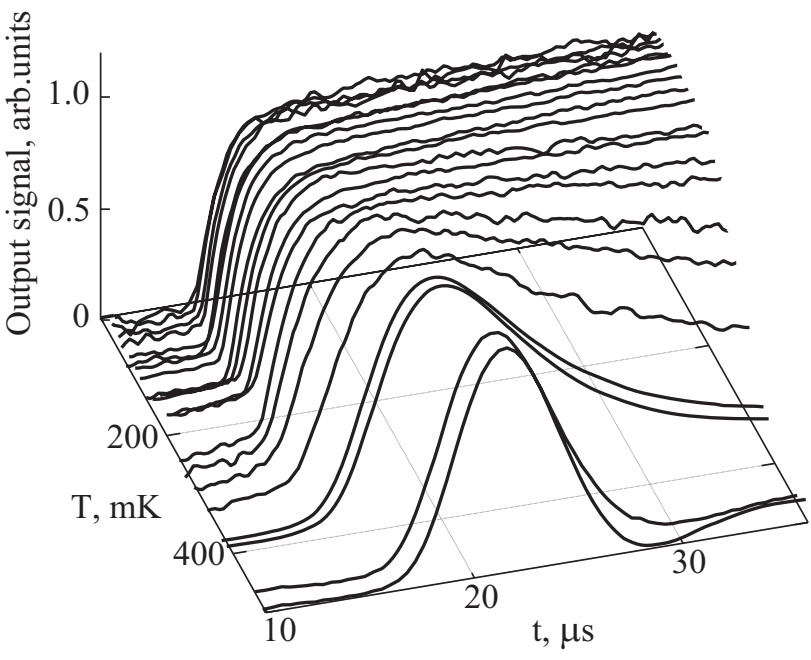

Fig. 2. Heat pulse propagation across solid ${ }^{4} \mathrm{He}$ at 56 bar as detected by LRB. A short (pulse width $t_{p w}=0.5 \mu \mathrm{s}$ ) heat pulse is generated at the heater at $t=0$. The voltage induced at the bolometer is averaged and the peak amplitudes are normalized to unity at all temperatures. The electronic cross talks during $t<10 \mu$ s are not shown.

over [21] from the second sound propagation in the normal solid ${ }^{4} \mathrm{He}$ in the high temperature range to ballistic transverse (see below for arguments behind this identification) phonon propagation in the low temperature range. Our expectation, as introduced in Sec. 1, that heat pulses propagate as second sound $[21,25,26]$ in the high temperature region $T>350 \mathrm{mK}$ in the normal solid ${ }^{4} \mathrm{He}$ range is qualitatively satisfied by our data in Fig. 2. Ackerman and Guyer [26] observed and analyzed their measurements of second sound propagation in solid ${ }^{4} \mathrm{He}$ by solving «hydrodynamics» of weakly interacting phonons. They fitted their observed response with a pulse shape analysis procedure (see Appendix E of [26]) and extracted $\tau_{n}$ as a function of temperature. Their fitting procedure, however, was successful only for the data taken from the sample chamber with a large aspect ratio which presumably reduced the contribution from the side wall refections to the net response [26]. We attempted to follow their analysis procedure but our data could not be fitted satisfactorily owing mainly to the presence of flat response in the time interval, $20 \mu \mathrm{s}<t<35 \mu \mathrm{s}$, as seen at lower temperatures in Fig. 2. It is likely that our bolometer response was strongly influenced in the problematic time range by the multiple reflections from the side walls of the sample chamber.

In the circumstance that the measured time response could not be fitted to the phonon gas model, we extracted the propagation velocity from the arrival time of the heat pulse front. Fig. 3 illustrates the manner of our determination of the propagation velocity. The upper panel is the bolometer response at $36 \mathrm{mK}$ taken from Fig. 2. A small 


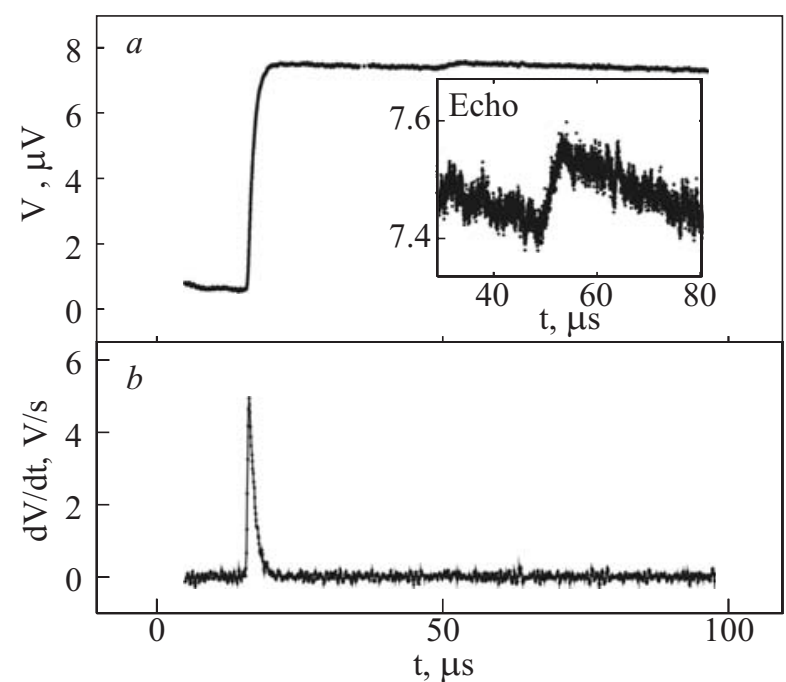

Fig. 3. Upper panel: induced voltage response of the bolometer to heat pulse applied at $t=0$ at $36 \mathrm{mK}$. The pressure of the solid is 56 bar. Inset is a magnified portion showing the echo which traverses over the distance $3 L$. Lower panel: the time derivative of the detected signal in the upper panel. The electronic cross talk signal prior to $5 \mu$ s is not shown.

echo of the heat pulse is observed on top of the «background» around $t \sim 44 \mu$ s (see inset of Fig. 3). The sharp leading edge of the heat pulse arrives at $t \sim 14 \mu$ s. The transit time for the sharp leading edge to reach the bolometer was determined from the peak position $t_{0}$ of the time derivative (see lower panel in Fig. 3,b). The propagation velocity (see Fig. 5) is evaluated simply as $L / t_{0}$. The echo location is consistent with the pulse traversing back to heater substrate surface and returning upon reflection.

There are three possible sound propagation modes in hep solid ${ }^{4} \mathrm{He}$, one longitudinal and two («slow» and «fast») transverse [42]. The observed propagation mode is argued as the slow transverse mode as follows. The expected longitudinal mode propagation velocity at 56 bar is at least $530 \mathrm{~m} / \mathrm{s}$. The propagation velocity (see Figs. 4 and 5) is much smaller than the longitudinal mode. Fig. 4 shows the observed pressure dependence of the propagation velocity plotted as a function of Debye temperature [43]. It is remarkable that the measured velocity varies linearly with the Debye temperature for all samples between 25.3 and 56 bar except for one. This linearity indicates that those samples apparently grew with similar crystal orientations. Comparison of the higher velocity $(251 \mathrm{~m} / \mathrm{s})$ point near the melting pressure in Fig. 4 with Fig. 11 of Ref. 42 shows that the observed mode is the slow transverse mode and the «effective» angle $\phi$ of the propagation direction with respect to $c$-axis for these samples is $(19 \pm 3)^{\circ}$. Comparison again with Fig. 11 of Ref. 42 shows that $\phi$ of the exceptional sample near the melting pressure with lower propagation velocity $(225 \mathrm{~m} / \mathrm{s})$ is

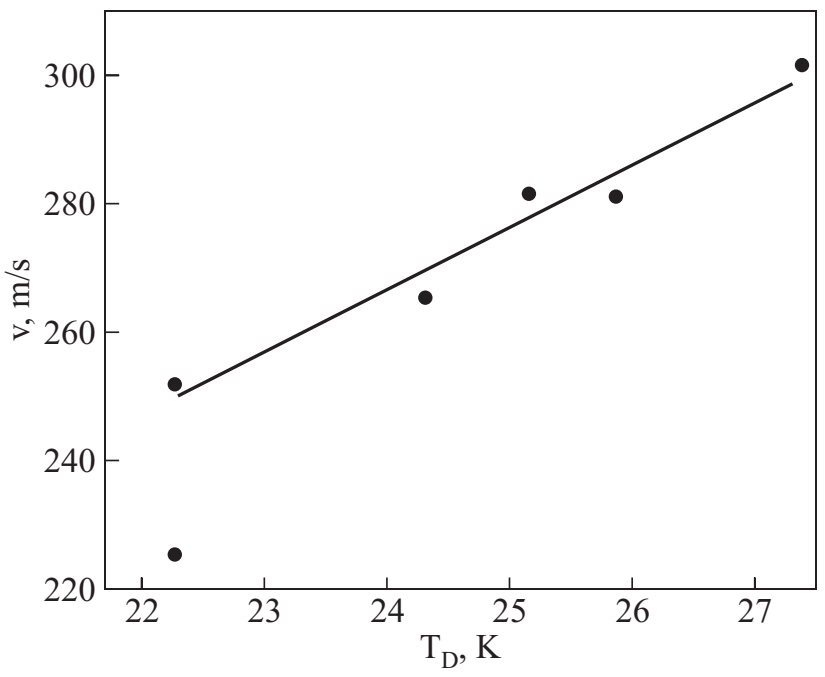

Fig. 4. Pressure dependence of measured propagation velocity. Pressure variation is expressed as a function of Debye temperature. Except for one data point near the melting pressure, all other points lie along a straight line (shown as guide to the eye). All samples were made with the blocked capillary method. See text for a discussion of the sample which does not follow the linear trend.

$(90 \pm 5)^{\circ}$. The minimum propagation velocity of the fast transverse mode for any orientation angle $\phi$ is $260 \mathrm{~m} / \mathrm{s}$. This minimum is outside the uncertainty of our measurement. Thus, it is concluded that the predominant propagation mode in our experiment is the slow transverse mode. It should be noted that others [21,31] found that trans-

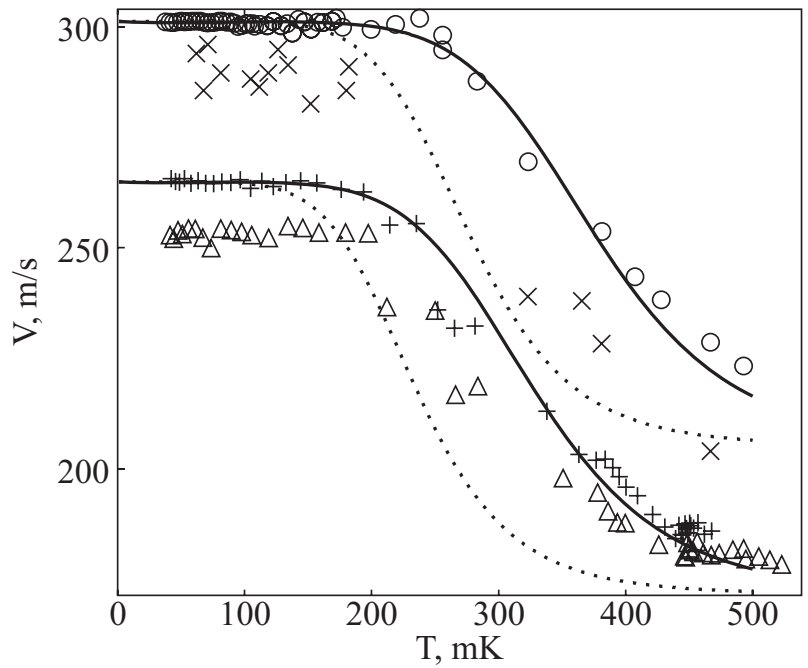

Fig. 5. The temperature dependence of the transverse velocity at 37 (pluses and triangles) and 56 (circles and crosses) bar. Pluses and circles were the velocities evaluated from the initial pulse arrival time. Triangles and crosses are the velocities evaluated from the echo signals (see Fig. 3). Solid lines are fits to the initial arrival velocity using the dispersion relation given by Eq. (1). Dotted lines show the propagation velocity expected for the echo signal based on the normal phonon collision time $\tau_{n}$ obtained from fitting the first arrival. 
verse mode was the much better excited than the longitudinal mode in solid ${ }^{4} \mathrm{He}$ by heat generators. Efforts were made to detect the longitudinal mode, but no clear evidence for it could be seen in our experiments.

Temperature dependence of the slow transverse mode propagation velocity evaluated in the manner described above are shown in Fig. 5 for solid samples at 37 and 56 bar. Our measurements are the first of this kind carried out down to very low temperatures at pressures higher than the melting pressure. Velocities evaluated from both the initial signal arrival and the (less accurate) echo signal are shown.

The data clearly show the crossover in velocity from ballistic to second sound propagation as temperature increases. We analyze the data using the dispersion relation given by Eq. (1) with two parameters: $\omega$ and $\tau_{n}$. The ballistic phonon propagation velocity $v_{\mathrm{I}}$ is set by the experiment in the low temperature limit of the measured velocity $(265 \mathrm{~m} / \mathrm{s}$ at $37 \mathrm{bar}$ and $301 \mathrm{~m} / \mathrm{s}$ at $56 \mathrm{bar})$. The second sound velocity is evaluated according to $v_{\mathrm{II}}=v_{D} / \sqrt{3}$, where $v_{D}$ is the Debye velocity, $v_{D}=k_{B} T_{D} / \hbar\left(6 \pi^{2} N\right)^{1 / 3}$ ( $181 \mathrm{~m} / \mathrm{s}$ at 37 bar and $201 \mathrm{~m} / \mathrm{s}$ at 56 bar) [26], where $T_{D}$ is the Debye temperature [43]. Choice of the characteristic frequency $\omega$ is not so obvious. Phonon propagation is ballistic when $\omega^{-1}$ is short compared to $\tau_{n}$ during the phonon transit from the heater to the detector. Thus, it appears reasonable to approximate $\omega^{-1}$ for the first arrival signal as the transit time: $\omega=2 \pi v_{\text {II }} / L$. With these constraints on these three parameters, best fits to the data were searched for with the normal relaxation time in the power law form, $\tau_{n}=b T^{-p}$, with $b$ and $p$ as adjustable parameters. Reasonable fits to $v_{d}$ (lines in Fig. 3) are obtained with the values of $\tau_{n} T^{3.3} \sim 8 \cdot 10^{-8} \mathrm{~s} \cdot \mathrm{K}^{3.3}$ at $37 \mathrm{bar}$, and $\tau_{n} T^{3.6} \sim 8 \cdot 10^{-8} \mathrm{~s} \cdot \mathrm{K}^{3.6}$ at 56 bar. These values turn out to be close to that given in Ref. 8 at 25 bar. Based on our approximation, $\omega$ for the echo signal is reduced by a factor of 3 from that of the initial arrival signal. Dotted lines in Fig. 5 show the expected dispersions for the echo signals with the values of $\tau_{n}$ taken from fitting the initial arrival signals at the two pressures. There is qualitative agreement, but the echo signal data are not sufficiently precise for critical comparison.

According to the hydrodynamic theory (see Eq. (4)), the transverse sound propagation velocity should increase if solid ${ }^{4} \mathrm{He}$ is a supersolid. Thus, it is of importance to measure the temperature dependence in detail in the low temperature limit. Our experimental data below $150 \mathrm{mK}$ are shown in Fig. 6 as deviations from the measured velocity $\left(v_{0}\right)$ at $200 \mathrm{mK}$. It can be seen that the measured ballistic phonon propagation velocities are constant within $\pm 0.15 \%$ scatter below $100 \mathrm{mK}$ at both pressure shown in Fig. 5. The dotted and dash-dotted lines show the expected temperature dependence from Eq. (4) at 53.6 and $30 \mathrm{bar}$, respectively, where the superfluid fractions

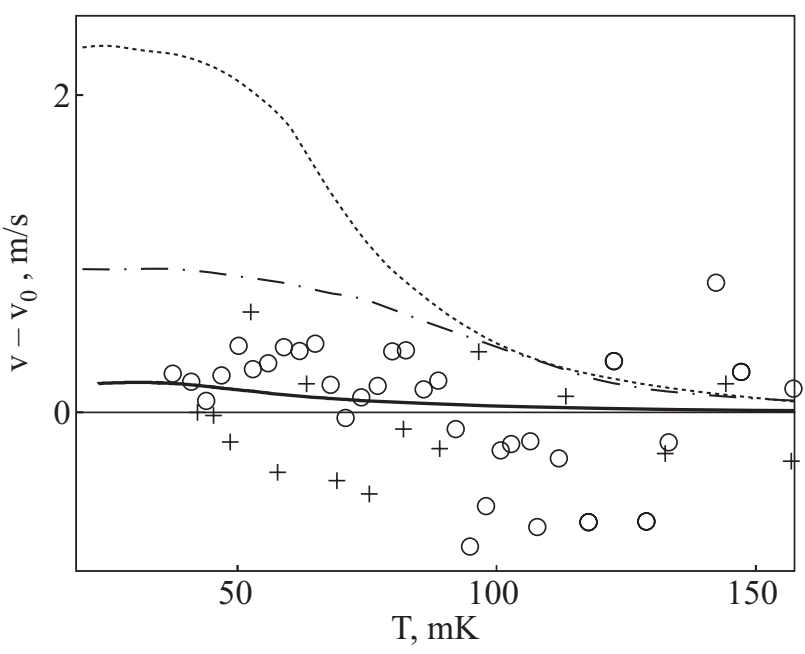

Fig. 6. Change in ballistic phonon propagation velocity at 56 bar. The reference velocity $v_{0}$ is that measured at $200 \mathrm{mK}$. Symbols have the same meaning as in Fig. 5. Dotted line and dash-line dot show the expected changes in the transverse velocity according to Eq. (4) with $\rho_{s} / \rho$ taken from [38] at 53.6 and 30 bar, respectively. The solid line shows the expected velocity when the superfluid fraction is taken from our own torsional oscillator experiment [44] with cylindrical sample cell.

are taken from those measured in the annular cell in Ref. 38. It is clear that the data and the expected temperature dependence do not match. If the much smaller superfluid density measured in our own [44] torsional oscillator with a cylindrical cell is assumed, then the expected temperature dependence (see solid line) is well within the scatter and the data is not inconsistent with the expectation. Since the cylindrical geometry of our torsional oscillator is closer to the present heat pulse experiment chamber, it is likely that this superfluid density is more appropriate.

The relation, if any, between the reduction in superfluid density as seen in critical velocity effects in the torsional oscillator experiments $[5,44]$ and the excitation energy in our heat pulse generator is important to investigate. Kim and Chan [38] found that the measured superfluid fraction was dependent on the amplitudes of the solid ${ }^{4} \mathrm{He}$ velocity: the fraction was nearly constant up to about $10 \mu \mathrm{m} / \mathrm{s}$ and it decreased at amplitudes higher than this. Superfluidity might even be totally destroyed beyond some critical velocity as yet undetermined. To search for velocity-dependent effect in our experiment, we measured the transverse ballistic phonon velocity as a function of the input pulse power at $40 \mathrm{mK}$ [40]. If the superfluid density increases as the input pulse power is decreased, the phonon velocity is expected to increase according to Eq. (4). The measured phonon velocity was constant within $\pm 0.1 \%$ at all input pulse energy higher than $1.2 \mathrm{~nJ} /$ pulse. When the pulse energy was less than $1.2 \mathrm{~nJ} /$ pulse, the propagation velocity could be deter- 
mined only within $\pm 0.8 \%$ owing to the increased scatter in data. We have also measured the pulse power dependence of the amplitude $\Delta T$ at $t \sim 40 \mu$ s to search for possible deviation at some critical pulse power. The measured amplitude $\Delta T$ increased smoothly, and no clear critical pulse power effect was observed.

\subsection{Search for fourth sound in solid ${ }^{4} \mathrm{He}$ below $200 \mathrm{mK}$}

In this Section we present our search for fourth sound propagation. If a fourth sound propagation is found, it becomes a new important evidence for supersolid state in ${ }^{4} \mathrm{He}$. Our search methods and the sensitivity limits of our bolometer detectors are given here. Attempts to produced fourth sound by changing the quality of solid are described. When an anomalous behavior is observed in a solid sample, we check to see if the same behavior is observed when the solid is replaced by superfluid. In brief, we have not yet succeeded in observing any clear signature which could be identified as fourth sound propagation in any of our solid ${ }^{4} \mathrm{He}$ samples.

According to the two fluid model hydrodynamics $[2,15]$ of supersolid, fourth sound velocity is given by Eq. (3). Taking $\rho_{s} / \rho \sim 0.01$ and $C_{1} \sim 500 \mathrm{~m} / \mathrm{s}, C_{4}$ at low temperatures $(T<30 \mathrm{mK}$ ) would be $\sim 50 \mathrm{~m} / \mathrm{s}$ (corresponding to the transit time of $\sim 100 \mu$ s in our cell). A fourth sound propagation should be accompanied by a temperature oscillation [34]. If our heat pulse generator did launch a fourth sound wave, an associated temperature disturbance should propagate in some characteristic manner. Depending on the origin of superfluidity such as particle exchange [3], grain boundaries [45], glassy supersolid [46] and screw dislocations [47], the propagation velocity of fourth sound is expected to reflect the nature of the origin. We searched for some such fourth sound-like signature by measuring the bolometer response in time intervals up to $40 \mathrm{~ms}$ following the input heat pulse (arrival time of $1 \mathrm{~ms}$ corresponds to the propagation velocity of $4 \mathrm{~m} / \mathrm{s}$ ) as a function of temperature between 40 and $250 \mathrm{mK}$. Careful searches in at least three solid samples revealed no identifiable signature of fourth sound in the measured temporal response.

Our search for fourth sound propagation using LRB in the 56 bar sample is summarized in Fig. 7 showing the normalized averaged detected signals as functions of time $(t<10 \mathrm{~ms})$ and temperature $(30<T<400 \mathrm{mK})$. The generator heater was driven with $1.6 \mathrm{~nJ}$ of energy per pulse $(0.5 \mu$ s wide) at a low repetition frequency of $19 \mathrm{~Hz}$ to avoid overheating. The initial rapid rise corresponds to the arrival of transverse sound already described (see Figs. 2 and 3). Subsequent to the rapid rise, the signal decreases with time dependence close to exponential decay. To look for any small hidden features that might systematically shift as the temperature was changed, this exponential «background» was subtracted from the signal. No

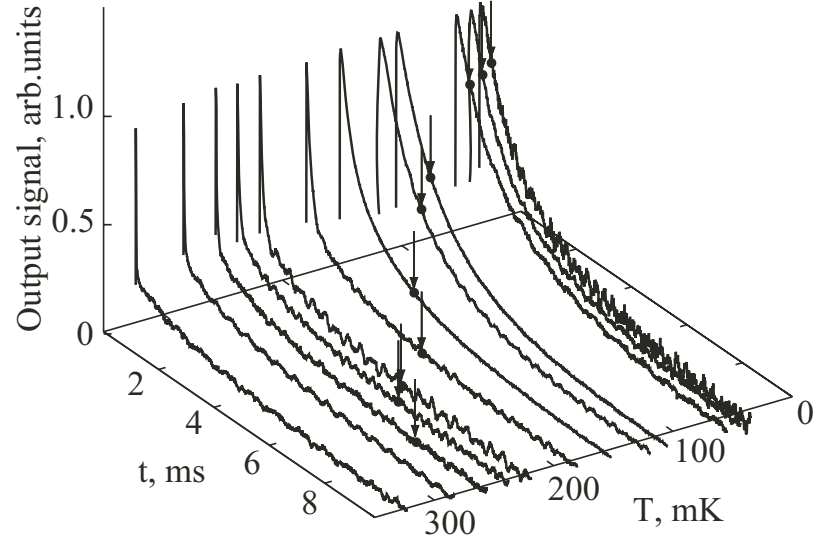

Fig. 7. The temporal development of the detected signal using LRB in search of fourth sound propagation. The long tail portion of the response is highlighted where the fourth sound is expected to influence and exhibit some signature. The arrows indicate the times at which fourth sound signal is expected (see text).

temperature dependent feature could be found in the difference signals. The arrows in Fig. 7 indicate the times at which fourth sound signal is expected from Eq. (3) with the superfluid fraction being taken from our torsional oscillator experiment [44].

Since the single strip LRB failed to detect fourth sound, a new bolometer (HRB) was fabricated to increase the sensitivity by an order of magnitude. See Sec. 3 for details of the bolometers. The averaged detected signals from HRB converted to temperature deviations at selected temperatures are shown in Fig. 8. The generator heater was driven with $9 \mathrm{~nJ}$ per pulse ( $10 \mu$ s wide) at again $19 \mathrm{~Hz}$ repetition frequency to avoid overheating. Owing to the larger resistance in the bolometer, the time response to the initial arrival of the transverse sound was poorer than LRB. The slower time response of the HRB should not have diminished sensitivity for detecting fourth sound at low frequencies. To improve the signal to noise ratio, the total averaging time was increased to as much as 10 hours during which the temperature was maintained constant within $0.1 \mathrm{mK}$. Careful scrutiny for any systematically developing deviation in the signals from «smooth» variation did not reveal any fourth sound like signature. If there were a fourth sound-like propagation in solid ${ }^{4} \mathrm{He}$, we would expect some characteristic signature on top of the background of the transverse sound signal. No signature has been observed within a temperature excursion of about $\Delta T=5 \mu \mathrm{K}$. The dots in Fig. 8 again represent the times at which fourth sound signals are expected.

$\Delta T$ at the peak response in Fig. 8 became higher at lower temperatures and was approximately inversely proportional to the heat capacity of solid ${ }^{4} \mathrm{He}$. This indicates that the solid sample comes to an equilibrium when the peak temperature is reached after application of heat 


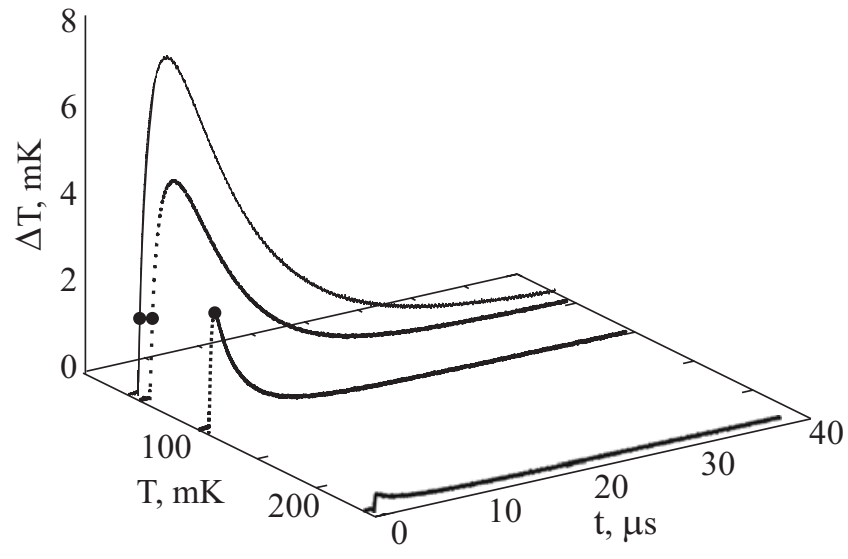

Fig. 8. Temporal development of the detected signal using HRB in search of fourth sound propagation at selected temperatures. The dots indicate the times at which fourth sound signal is expected (see text). The sample solid was formed relatively slowly in about three hours at 38 bar.

pulse. $\Delta T$ relaxed towards the ambient mixing chamber temperature. The relaxation time of this process became longer at temperatures below $80 \mathrm{mK}$. This relaxation process was not peculiar to solid ${ }^{4} \mathrm{He}$, since a similar temperature dependence of relaxation time was observed if the solid was replaced by liquid at 24.5 bar (see next Section for more discussion of relaxation time).

It is possible that the above null results are the consequence of degradation or possibly destruction of superfluidity by exceeding some critical heat input to the generator. To alleviate such an effect, the heat input to the generator was decreased by a factor of 70 and the search was repeated. In addition, to attempt to increase the superfluid fraction [8], the solid sample was made in a fast cooling process in which ${ }^{4} \mathrm{He}$ was frozen over several minutes. (See below for more detailed study of sample preparation procedure.) Figure 9 shows the responses of HRB in this search with low heat input level in the fast cooling sample.The input energy was $0.13 \mathrm{~nJ} / \mathrm{pulse}$, and the response was taken at each regulated temperature by averaging $5 \cdot 10^{5}$ repetitions. Similar measurements in the slow cooling sample were carried out and the input pulse energy was decreased down to $0.014 \mathrm{~nJ}$, but no signal with characteristics of fourth sound could be decerned.

The relationship between the generated superfluid velocity and the input heat energy is uncertain. However, an estimate on the temperature variation generated by superflow might be made in analogy with sound propagations in He II as $\delta T / T \approx v_{s} / C_{4}$, where $v_{s}$ here is taken as a critical velocity. If the superfluid fraction is taken as $1(0.1) \%$ and a critical velocity as $10(500) \mu \mathrm{m} / \mathrm{s}$ from Refs. $38(44), \delta T / T \approx 2 \cdot 10^{-7}\left(3 \cdot 10^{-5}\right)$. With our current bolometer, the limit on a detectable signature in temperature variation is $\Delta T \approx 5 \mu \mathrm{K}$ or $\Delta T / T \approx 10^{-4}$ at $T=$ $=50 \mathrm{mK}$. The HRB sensitivity is actually not sufficient to

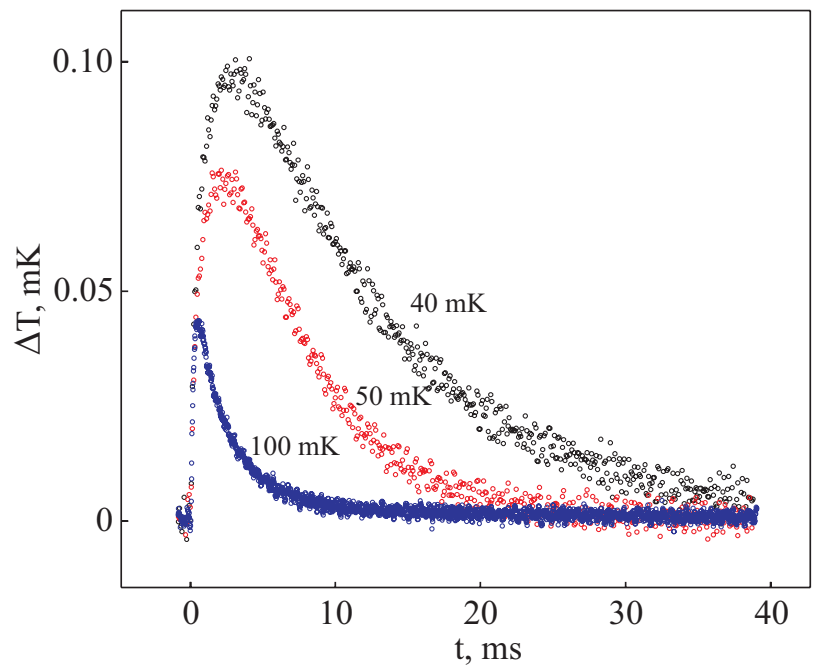

Fig. 9. Overall responses of the bolometer at 40,50 and $100 \mathrm{mK}$ in the fast cooling sample at 38 bar. The bolometer HRB with higher sensitivity was the detector. No likely signature for fourth sound propagation was observed.

detect this temperature variation. It must then be allowed that fourth sound in solid ${ }^{4} \mathrm{He}$ might NOT be detectable with our present bolometer. In this sense, our experiment does not definitively rule out fourth sound propagation in the supersolid state of ${ }^{4} \mathrm{He}$.

The torsional oscillator experiments by Rittner and Reppy $[8,48]$ and Kondo, et al. [49] showed that the solid ${ }^{4} \mathrm{He}$ sample preparation procedure played an important role in the observed NCRI fraction. To investigate the effect of sample preparation in our experiment, we formed different samples by changing the cooling rate during solidification, and compared the responses of the bolometer in each sample. Figure 10 shows the HRB responses in two solid samples formed with different cooling rates. One sample («slow cooling») was formed in ten hours to cool from liquid phase at $3 \mathrm{~K}$ and 73 bar to solid at $1 \mathrm{~K}$. The other («fast cooling») was cooled down in 30 minutes to $1 \mathrm{~K}$. The final pressure in both samples was 38 bar. The input heat pulse width $t_{p w}$ was $10 \mu$ and the input energy was $0.36 \mathrm{~nJ} /$ pulse. There are interesting difference and similarity between the two samples. In the slow cooling sample, the initial sharp rise in temperature represents the arrival of transverse phonons as observed with the LRB. If the bolometer response was not limited by the RC electronics time constant $(\sim 0.1 \mathrm{~ms})$, it would reach the maximum within $10 \mu$ s after the transverse phonon arrival as seen in Fig. 3. The long elapsed time interval to reach the maximum clearly showed that the initial response was limited by the RC time constant. In the fast cooling sample, on the other hand, the elapsed time ( $3 \mathrm{~ms}$ at $40 \mathrm{mK}$ ) to reach the maximum became much longer and was not limited by the RC time constant of the bolometer circuitry. In the fast cooling sample, the initial thermalization within 


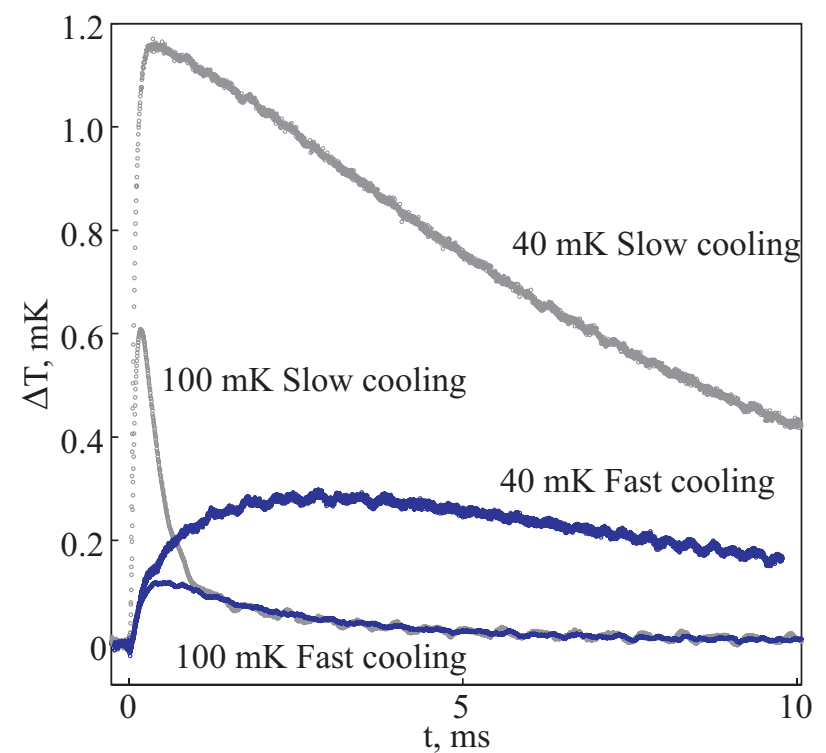

Fig. 10. Time response of HRB with different sample preparation procedures: dots and crosses for «fast cooling» and «slow cooling» sample, respectively (see text), at 38 bar. Responses of the two samples are compared at 40 and $100 \mathrm{mK}$.

the sample required much longer time than the slow cooling sample. This difference in the initial response resulted almost certainly from the difference in sample preparation procedure resulting in different «quality» solids. In contrast to the difference in the initial response, the ensuing final decay responses towards the ambient temperature were similar in both samples. At $t>3$ (1) $\mathrm{ms}$ at 40 (100) $\mathrm{mK}$, the signal decayed to zero exponentially, and this decay time constant (10 (2) $\mathrm{ms}$ at $40(100) \mathrm{mK})$ did not depend on sample preparation procedure. The final decay process was likely the energy flow from the solid ${ }^{4} \mathrm{He}$ sample to the copper walls of the container via the thermal boundary resistance. The thermal boundary resistance did not apparently depend on the sample preparation procedure. Independence from sample preparation procedure is reasonable since the dominant thermal resistance occurs at the boundary between the packed copper powder heat exchanger and helium. More data and analysis of these time constants are given in the next Section.

Above observations show that the the response by solid ${ }^{4} \mathrm{He}$ to heat pulse can be used to distinguish fast cooling (solidification within several tens of minutes) from slow cooling (solidification over three hours) samples. When the slow-cooling sample was «annealed» by warming and keeping the temperature between 1.2 and 1.4 for ten hours did not change the response. It appears that the slow-cooling sample was already annealed. Heat pulse measurement may act as a useful tool in differentiating «good» from «bad» quality samples in the study of supersolid phenomena. It would be interesting to carry out simultaneous measurements of heat pulse and tor- sional oscillation to see if the quality of sample can be correlated with the NCRI fraction. A simultaneous measurement with solid pressure would also be interesting to see if the observed glassy behavior [50] is correlated with longer thermal response relaxation.

According to an alternate non-superfluidity-based theory [51], the observed NCRI behavior [37] is related to a glassy state in ${ }^{4} \mathrm{He}$ crystals induced by dislocation tunneling systems. In a crystal of dislocation tunneling systems, after the heat pulse is applied to the crystal, it is initially absorbed by the phonon bath and subsequently transferred to the tunneling systems causing their repopulation. This process would result initially in an «overshoot» in temperature rise and then settles to a lower temperature [52]. Presumably, these transient changes occur with characteristic time constants. The crystal eventually returns to the ambient temperature as the heat dissipates into the mixing chamber heat bath via thermal boundary resistance as discussed above. Such time development was not seen in our bolometer responses (see, for example, Fig. 9). An analysis of the dynamic thermal response is presented in the next section.

\subsection{Dynamic thermal response}

The entire time response subsequent to the arrival of transverse phonons such as those shown in Figs. 9 and 10 can be represented by the difference of two exponentials, $\Delta T_{0}\left[\mathrm{e}^{-t / \tau_{2}}-\mathrm{e}^{-t / \tau_{1}}\right]$. This formula describes the processes of internal thermalization within the solid sample occurring with a time constant $\tau_{1}$ and simultaneous energy flow out of the sample to the thermal bath (mixing chamber) occurring with another time constant $\tau_{2}$. Fitted time constants are shown in Figs. 11 and 12 for fast and slow cooling samples. The time constant $\tau_{1}$ was measured only

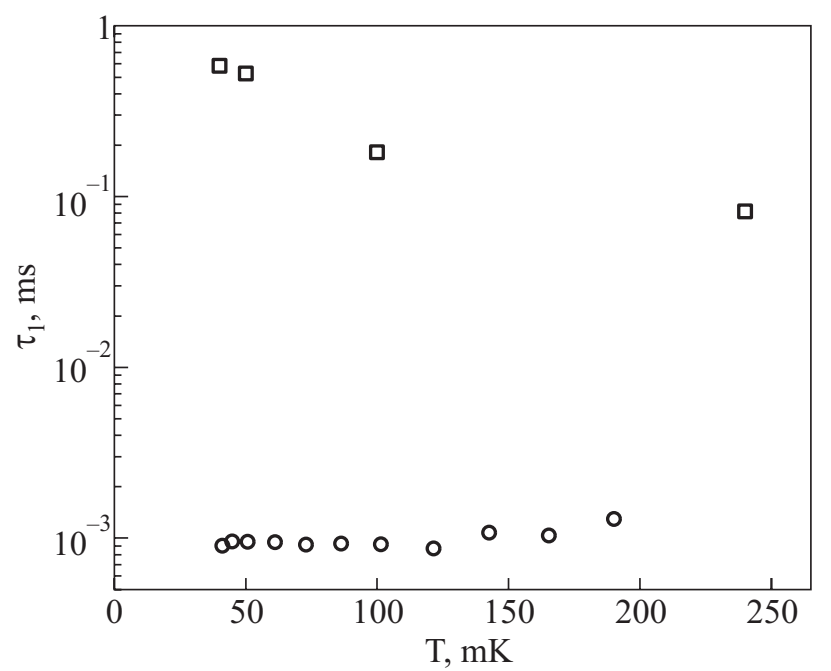

Fig. 11. Fitted $\tau_{1}$, the thermalization time within solid ${ }^{4} \mathrm{He}$ (see text), as a function of temperature. Squares are from «dirty» fast-cooling sample measured with HRB and circles from «clean» slow cooling sample measured with LRB. 


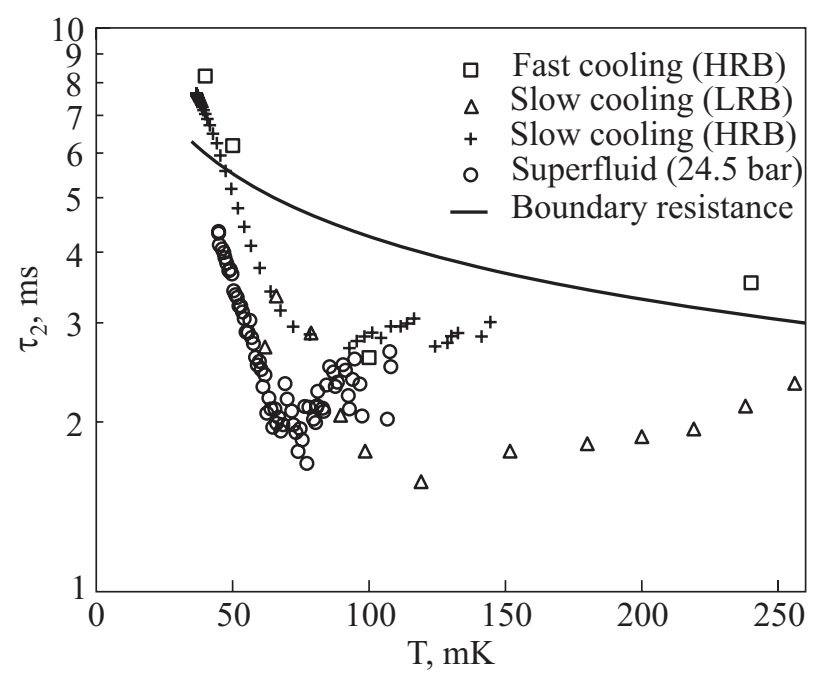

Fig. 12. Fitted $\tau_{2}$, thermalization time to the ambient temperature (see text), as a function of temperature for «dirty» fastcooling and «clean» annealed solid samples both at 38 bar. Note that the fast cooling sample was measured with HRB and the slow cooling one with LRB.

with HRB in the fast cooling sample, but it is expected to be same if measured with LRB: $\tau_{1}$ could not be measured in the slow cooling sample by HRB owing to its relatively long RC electronic time constant $(\sim 0.1 \mathrm{~ms})$. Note that $\tau_{1}$ in the slow cooling sample is more than two orders of magnitude smaller that that of the fast cooling sample. The difference presumably arises from the varying «quality» of the solid samples.

The thermalization time within the solid ${ }^{4} \mathrm{He}$ sample at low temperatures may be estimated by $r^{2} / D$ where $r$ is the size of the sample and $D=K / \Gamma$ is the thermal diffusivity, $K=\Gamma c l / 3$ the thermal conductivity, $\Gamma$ the heat capacity per unit volume, $c$ the phonon velocity and $l$ the phonon mean free path. The thermalization time is then $3 r^{2} / \mathrm{cl}$. In the temperature range, where $c$ is constant, the thermalization time is proportional to $l^{-1}$. Since the mean free path is likely to be determined by the grain size, the relaxation time is expected to be independent of temperature. It can be seen from Fig. 11 that $\tau_{1}$ in the slow cooling sample is indeed independent of temperature. If we take $r \sim 5 \mathrm{~mm}$ (from the cell size) and $c=400 \mathrm{~m} / \mathrm{s}$, the above analysis would imply the mean free path in the slow cooling sample to be roughly $20 \mathrm{~mm}$. The long evaluated mean free path indicates that the slow cooling sample is a high quality solid. On the other hand, $\tau_{1}$ in the fast cooling sample is much greater and the mean free path evaluated at $200 \mathrm{mK}$ is $\sim 0.2 \mathrm{~mm}$. Furthermore, it shows a faster increase below $100 \mathrm{mK}$. This unexpected temperature dependence is not due to rapid change in the bolometer response time. The increase in $\tau_{1}$ occurs in the same temperature range as the supersolidity range seen in torsional oscillator experiments. An increase in $\tau_{1}$ could result from a decrease in the phonon mean free path $l$. It is difficult to see why $l$ would decrease as the temperature is lowered in a supersolid state. In their $d c$ measurement of thermal conductivity by Armstrong and Greenberg [53], no anomaly in thermal conductivity was observed in $3 \mathrm{~mm}$ diameter solid ${ }^{4} \mathrm{He}$ samples in the same temperature range as ours. The apparent decrease in thermal conductivity or mean free path in our pulse measurement of fast cooling sample below $100 \mathrm{mK}$ might possibly be a frequency dependent effect. Further studies are needed to see if the increase in $\tau_{1}$ is related to supersolidity.

Comparing Figs. 11 and 12 shows that $\tau_{1}<\tau_{2}$. This indicates that the temperature of the solid sample may be considered uniform during the decay after reaching the peak response in Fig. 10. It is interesting to note that $\tau_{2}$ is independent of the quality of the solid sample and that it is not affected significantly by replacing solid with $\mathrm{He}$ II at 24.5 bar. It is natural to consider $\tau_{2}$ as the thermal equilibration time between the solid ${ }^{4} \mathrm{He}$ sample and the mixing chamber. If this identification is appropriate, it would be related to the thermal boundary resistance and heat capacity $C$ by $\tau_{2}=R_{B} C$, where $R_{B}$ is the thermal boundary resistance. The thermal boundary resistance between copper and solid ${ }^{4} \mathrm{He}$ is taken as [54] 2.6.10 ${ }^{-3} T^{-3.7} / A$ $\mathrm{K}^{4.7} \mathrm{~cm}^{2} / \mathrm{J}$, where $A$ is the contact surface area. The solid line in Fig. 12 shows the calculated time constant when $A$ is adjusted to $90 \mathrm{~cm}^{2}$. The boundary area is dominated by the packed copper powder in the heat exchanger and the adjusted surface area is not an unreasonable estimate for its surface area. The calculated $\tau_{2}$ captures the qualitative but not the detailed temperature dependence. Since the thermal boundary resistance at the copper/solid ${ }^{4} \mathrm{He}$ interface is smaller than at the copper/ He II interface [54] by a factor of seven, $\tau_{2}$ is expected to be smaller when the cell is filled with solid ${ }^{4} \mathrm{He}$ (the heat capacity of solid ${ }^{4} \mathrm{He}$ is greater than that of He II by only a factor of two). This discrepancy has not been resolved. Further studies are also needed to clarify the origin of the relaxation time $\tau_{2}$.

\subsection{Why is fourth sound signal absent?}

We began our search for fourth sound propagation in solid ${ }^{4} \mathrm{He}$ in the temperature range where the non-classical rotational inertia has been observed. Our search so far has turned out negative. There are a number of difficulties which might render fourth sound undetectable in our experiments. We explore some of these here and discuss remedies and improvements.

The effect of critical velocity has been already mentioned above. In order for our bolometer sensors to be sensitive to the presence of fourth sound, the heat pulse generator must of course produce detectable fourth sound signal. It is possible that the critical velocity is exceeded by our heat pulse generator resulting in destruction or severe diminishment of superfluidity. It appears that the 
sensitivity of our bolometer must be improved (see above) by at least an order of magnitude. It was found that annealed samples showed much smaller NCRI fraction than those «freshly» grown [8]. Considering that the samples in larger cells such as ours show small NCRI fractions, it might be fruitful to search for fourth sound in cells with larger surface area to volume ratio. Making fourth sound cells of annular shape with small gap between the outer or of packed powder are possible directions of our search.

Bolometric detection and heater generation may not be the optimal scheme for coupling to fourth sound. Fourth sound is expected to produce a thermal imprint, but it may be weak [34]. One might attempt acoustic generation/detection to search for a fourth sound signature. Search with this detection scheme, however, has also not been successful [55]. Of course, critical velocity effects must be considered with acoustic methods just as in our bolometric detection scheme. If the attenuation of fourth sound propagation is excessive, it would not be possible to detect its propagation by either bolometric or acoustic means. To our knowledge, there has been no theoretical prediction on the attenuation.

Another possibility is that supersolidity does not encompass superfluidity in the sense that its hydrodynamics has been formulated $[2,15,16]$. Then, fourth sound perhaps does not exist at all in the supersolid state. Interpretation of non-classical rotational inertia in terms of a glass state [51] and a two level system [56] would not lead to fourth sound propagation. The vortex liquid point of view [57] of supersolidity might not support fourth sound.

Path integral Monte Carlo studies have shown existence of superfluidity along grain boundaries in simulated quench-cooled «superglass» solid ${ }^{4} \mathrm{He}$ states [58]. Presumably, fourth sound propagation would be possible in such a system. Given that the "channel size» of the superfluid path along the grain boundaries is small, the conventional requirement of immobilizing the normal component for fourth sound propagation should be well satisfied. The tortuosity of the network of grain boundaries might be large and the measured propagation velocity might be reduced considerably by a corresponding index of refraction. The index of refraction is likely to be dependent on the details of the sample preparation procedure. It would be of interest to carry out theoretical studies of sound propagation in the superglass superfluid state.

\section{Conclusion}

The path-breaking torsional oscillator experiment by Kim and Chan [4] showed that solid ${ }^{4}$ He samples in simply or multiply connected geometries exhibited non-classical rotational inertia. The observed non-classical rotational inertia was interpreted as a long-sought evidence for superfluidity in solid ${ }^{4} \mathrm{He}$ at very low temperatures. The goal in our work is to search for a new experimental evidence for superfluidity in solid ${ }^{4} \mathrm{He}$. It is expected on theoretical grounds that there should emerge a new propagating sound mode in a supersolid state analogous to fourth sound sound in superfluid ${ }^{4} \mathrm{He}$. Detection of such fourth sound mode would provide a strong supporting evidence for superfluidity in solid ${ }^{4} \mathrm{He}$. We have attempted to generate and detect fourth sound in solid ${ }^{4}$ He samples grown in a cylindrical tube by the scheme of heat pulse and a titanium superconducting edge bolometer sensor. In agreement with earlier measurements by Narayanamurti and Dynes [21], we observed the crossover of transverse phonon propagation from the «second sound» regime in normal solid at high temperatures to the ballistic propagation regime below $200 \mathrm{mK}$. Our search for fourth-sound-like mode with slow propagation velocity and the expected characteristic temperature dependence has not uncovered any positive evidence. Searches were made at the lowest generator drive level to alleviate critical velocity effects which might inhibit fourth sound propagation. The sample quality was altered by varying the solid growth rate as well as annealing. The sample quality change could be detected by the observed thermal response. Changes in sample quality did not help in revealing fourth sound propagation. Searches for fourth sound was made at solid pressure between 25 and 56 bar. Possible reasons for why fourth sound propagation was not detected were discussed. Whether or not fourth sound propagation occurs in the supersolid state of ${ }^{4} \mathrm{He}$ remains an important issue in understanding supersolidity of ${ }^{4} \mathrm{He}$.

We are grateful to Wayne Saslow for stimulating discussions and communicating results prior to publication. We thank David Olaya, Misha Gershenson and Tony Clark for fabricating the titanium bolometers used in this work. Y.A. and H.K were supported by NSF through grants DMR0456862 and DMR0704120 and X.L. by NSF through grants DMR0207071 and DMR0706339. H.K. thanks Kavli Institute of Theoretical Physics and Pacific Institute of Theoretical Physics, where portions of this work were carried out.

1. M.W. Meisel, Physica B178, 121 (1992).

2. A.F. Andreev and I.M. Lifshitz, Zh. Eksp. Teor. Fiz. 56, 2057 (1969); Sov. Phys. JETP 29, 1107 (1969).

3. A.J. Leggett, Phys. Rev. Lett. 25, 1543 (1970).

4. E. Kim and M.H.W. Chan, Nature (London) 427, 225 (2004).

5. E. Kim and M.H.W. Chan, Science 305, 1941 (2004).

6. K. Shirahama, M. Kondo, S. Takada, and Y. Shibayama, Bull. Am. Phys. Soc. 51, 450 (2006).

7. A. Penzev, Y. Yasuta, and M. Kubota, J. Low Temp. Phys. 148, 677 (2007). 
8. A.S.C. Rittner and J.D. Reppy, Phys. Rev. Lett. 97, 165301 (2006).

9. J. Day, T. Herman, and J. Beamish, Phys. Rev. Lett. 95, 35301 (2005).

10. J. Day and J. Beamish, Phys. Rev. Lett. 96, 105304 (2006).

11. S. Sasaki, R. Ishiguro, F. Caupin, H. J. Maris, and S. Balibar, Science 313, 1098 (2006).

12. X. Lin, A. Clark, E. Kim, and M. Chan, in: APS Meeting Abstracts (2006), v. 51, p. 41010.

13. I.A. Todoshchenko, H. Alles, J. Bueno, H.J. Junes, A.Y. Parshin, and V. Tsepelin, Phys. Rev. Lett. 97, 165302 (2006).

14. N. Prokof'ev, ArXiv:cond-mat/0612499.

15. W.M. Saslow, Phys. Rev. B15, 173 (1977).

16. M. Liu, Phys. Rev. B18, 1165 (1978).

17. S.J. Putterman, Superfluid Hydrodynamics, North-Holland, Amsterdam (1974).

18. D. Vollhardt and P. Wolfle, The Superfluid Phases of Helium-3, Taylor and Francis, London (1990).

19.R.J.V. Gutfeld and J.A.H. Nethercot, Phys. Rev. Lett. 12, 641 (1964).

20. B.A. Young, B. Cabrera, and A.T. Lee, Phys. Rev. Lett. 64, 2795 (1990).

21. V. Narayanamurti and R.C. Dynes, Phys. Rev. B12, 1731 (1975).

22. S. Balibar, H. Alles, and A.Y. Parshin, Rev. Mod. Phys. 77, 317 (2005).

23. J. Ward and J. Wilks, Philos. Mag. 42, 314 (1951).

24. C.C. Ackerman and J.W.C. Overton, Phys. Rev. Lett. 22, 764 (1969).

25. C.C. Ackerman, B. Bertman, H.A. Fairbank, and R.A. Guyer, Phys. Rev. Lett. 16, 789 (1966).

26. C.C. Ackerman and R. Guyer, Ann. Phys. 50, 128 (1968).

27. S.J. Rogers, Phys. Rev. B3, 1440 (1971).

28. H.E. Jackson, C.T. Walker, and T.F. McNelly, Phys. Rev. Lett. 25, 26 (1970).

29. T.F. McNelly, S.J. Rogers, D.J. Channin, R.J. Rollefson, W.M. Gouba, G.E. Schmidt, J.A. Krumhansl, and R.O. Pohl, Phys. Rev. Lett. 24, 100 (1970).

30. V. Narayanamurti and R.C. Dynes, Phys. Rev. Lett. 28, 1461 (1972).

31. J.M. Goodkind, Phys. Rev. Lett. 89, 95301 (2002).

32. P.-C. Ho, I.P. Bindloss, and J.M. Goodkind, J. Low Temp. Phys. 109, 409 (1997).

33. W.M. Saslow, Phys. Rev. B71, 092502 (2005).

34. W.M. Saslow, Private Communication.

35. A.W. Yanof and J.D. Reppy, Phys. Rev. Lett. 33, 631 (1974).
36. H. Kojima, D.N. Paulson, and J.C. Wheatley, Phys. Rev. Lett. 32, 141 (1974).

37. E. Kim and M.H.W. Chan, J. Low Temp. Phys. 138, 859 (2005).

38. E. Kim and M.H.W. Chan, Phys. Rev. Lett. 97, 115302 (2006).

39. A.T. Dorsey, P.M. Goldbart, and J. Toner, Phys. Rev. Lett. 96, 055301 (2006).

40. Y. Aoki and H. Kojima, J. Low Temp. Phys. 148, 659 (2006).

41. Y. Aoki and H. Kojima, AIP Conference Proceedings 850, 1629 (2006).

42. R.H. Crepeau, O. Heybey, D.M. Lee, and S.A. Strauss, Phys. Rev. A3, 1162 (1971).

43. D.O. Edwards and R.C. Pandorf, Phys. Rev. 140, A816 (1965).

44. Y. Aoki, J.C. Graves, and H. Kojima, Phys. Rev. Lett. 99, 015301 (2007).

45. L. Pollet, M. Boninsegni, A.B. Kuklov, N.V. Prokof'ev, B.V. Svistunov, and M. Troyer, Phys. Rev. Lett. 98, 135301 (2007).

46. M. Boninsegni, N. Prokof'ev, and B. Svistunov, Phys. Rev. Lett. 96, 105301 (2006).

47. M. Boninsegni, A.B. Kuklov, L. Pollet, N.V. Prokof'ev, B.V. Svistunov, and M. Troyer, Phys. Rev. Lett. 99, 035301 (2007).

48. A.S.C. Rittner and J.D. Reppy, Phys. Rev. Lett. 98, 175302 (2007).

49. M. Kondo, S. Takada, Y. Shibayama, and K. Shirahama, J. Low Temp. Phys. 148, 695 (2007).

50. V.N. Grigorev, V.A. Maidanov, V.Y. Rubanskii, S.P. Rubets, E.Y. Rudavskii, A.S. Rybalko, Y.V. Syrnikov, and V.A. Tikhii, ArXiv:cond-mat/0702133v2.

51. Z. Nussinov, A.V. Balatsky, M.J. Graf, and S.A. Trugman, Phys. Rev. B76, 014530 (2007).

52. A.V. Balatsky, M.J. Graf, Z. Nussinov, and S.A. Trugman, Phys. Rev. B75, 94201 (2007).

53. G.A. Armstrong, A.A. Helmy, and A.S. Greenberg, Phys. Rev. B20, 1061 (1979).

54. F. Pobell, Matter and Methods at Low Temperatures, Springer-Verlag, Berlin (1996).

55. N. Mulders, Private Communication.

56. A.F. Andreev, ArXiv:cond-mat/0700571.

57. P.W. Anderson, Nat. Phys. 3, 160 (2007).

58. M. Boninsegni, N. Prokof'ev, and B. Svistunov, Phys. Rev. Lett. 96, 105301 (2006). 